\title{
Probing long-lived dark excitons in self-assembled quantum dots
}

Johansen, Jeppe; Julsgaard, Brian; Stobbe, Søren; Hvam, Jørn Märcher; Lodahl, Peter

Published in:

Physical Review B Condensed Matter

Link to article, DOI:

10.1103/PhysRevB.81.081304

Publication date:

2010

Document Version

Publisher's PDF, also known as Version of record

Link back to DTU Orbit

Citation (APA):

Johansen, J., Julsgaard, B., Stobbe, S., Hvam, J. M., \& Lodahl, P. (2010). Probing long-lived dark excitons in self-assembled quantum dots. Physical Review B Condensed Matter, 81(8), 081304.

https://doi.org/10.1103/PhysRevB.81.081304

\section{General rights}

Copyright and moral rights for the publications made accessible in the public portal are retained by the authors and/or other copyright owners and it is a condition of accessing publications that users recognise and abide by the legal requirements associated with these rights.

- Users may download and print one copy of any publication from the public portal for the purpose of private study or research.

- You may not further distribute the material or use it for any profit-making activity or commercial gain

- You may freely distribute the URL identifying the publication in the public portal 


\title{
Probing long-lived dark excitons in self-assembled quantum dots
}

\author{
Jeppe Johansen, Brian Julsgaard, Søren Stobbe, Jørn M. Hvam, and Peter Lodahl* \\ DTU Fotonik, Department of Photonics Engineering, Technical University of Denmark, ${ }^{\dagger}$ \\ $\emptyset$ rsteds Plads 343, DK-2800 Kgs. Lyngby, Denmark
}

(Received 15 January 2010; published 17 February 2010)

\begin{abstract}
Long-lived dark exciton states are formed in self-assembled quantum dots due to the combination of the angular momentum of electrons and holes. The lifetime of dark excitons are determined by spin-flip processes that transfer dark excitons into radiative bright excitons. We employ time-resolved spontaneous emission measurements in a modified local density of optical states to unambiguously record the spin-flip rate. Pronounced variations in the spin-flip rate with the quantum dot emission energy are observed demonstrating that the exciton storage time can be extended by controlling the quantum dot size. The energy dependence is compared to a recent model from the literature, in which the spin flip is due to the combined action of short-range exchange interaction and acoustic phonons. We furthermore observe a pronounced enhancement of the spin-flip rate close to semiconductor-air interfaces, which illustrates the important role of interfaces for quantum dot based nanophotonic structures.
\end{abstract}

DOI: 10.1103/PhysRevB.81.081304

PACS number(s): 78.67.Hc, 72.25.Fe, 78.47.-p

Long-lived dark excitons confined to quantum dots (QDs) are interesting candidates for spin qubits ${ }^{1}$ that can be prepared and read out optically. Dark excitons are nonradiative in nature and recombine only after a phonon-mediated spinflip process, whereby a radiating bright exciton is formed. ${ }^{2,3}$ Exploring this recombination process is essential since it influences the performance of QD single-photon sources for quantum information processing ${ }^{4-6}$ as well as the quantumoptical properties of QDs (Ref. 7) and ultimately limits inhibition of spontaneous emission in photonic crystals. ${ }^{8} 9$

In a QD, the exciton spin lifetime is greatly extended compared to a bulk medium or a quantum well due to the discrete energy spectrum. Due to the inherent multiparticle nature of solid-state systems the processes determining the lifetime of exciton spins are complex, and fundamental insight has been lacking. The physical mechanism behind the decoherence associated with spin-flip processes is debated in the literature, ${ }^{10,11}$ and experimental tests have been lacking. Here we employ controlled modifications of the local density of optical states (LDOS) to determine the dark exciton spinflip rate from time-resolved spontaneous emission measurements. The LDOS is modified by placing QDs at controlled distances from a semiconductor-air interface, which was previously used to distinguish radiative and nonradiative recombination processes of bright excitons. ${ }^{12}$ Controlling the LDOS provides a powerful tool to obtain detailed insight into QD dynamics; thus cavity quantum electrodynamics was recently employed for QD spectroscopy in the regime of continuous pumping. ${ }^{13}$ We observe a surprising dependence of the spin-flip rate on the distance to the sample surface ranging several hundred nanometers and a sensitive dependence on the QD emission energy. The latter enables a way of extending the dark exciton lifetime by controlling the QD size. The energy dependence is compared in detail to theory and provides valuable insight on the exciton spin-flip mechanism in QDs. Our work is an important step toward a full understanding of the complex dynamics of quantum dots in nanophotonic structures and dark excitons are potentially useful for long-lived coherent storage applications.

Figure 1(a) illustrates the fine structure of the lowest ex- citon state for InAs/GaAs QDs. ${ }^{14}$ The exciton is formed from the conduction-band electron state (spin 1/2) and the heavyhole valence-band state (total angular momentum 3/2). As a result, four exciton states are formed. They are characterized by the projections of the total angular momentum onto the growth axis, which attain the values \pm 1 or \pm 2 for bright and dark excitons, respectively. The splitting between dark and bright energy levels $\Delta_{d b}$ is determined by the exchange coupling between electron and hole spins, and the bright-exciton levels are typically a few hundred $\mu \mathrm{eV}$ above the darkexciton levels. The bright excitons $|b\rangle$ decay to the ground state $|g\rangle$ (no excitons) by emission of a photon through a dipole-allowed transition with a rate $\gamma_{\text {rad }}$. Radiative transitions from dark excitons $|d\rangle$ are forbidden, but they can decay through a phonon mediated spin-flip process (rate $\gamma_{d b}$ ) transforming the dark exciton into a bright exciton. The rate of the reverse process is denoted $\gamma_{b d}$. Finally nonradiative recombination was proven to be significant for selfassembled QDs, ${ }^{12}$ i.e., bright and dark excitons can recombine nonradiatively with rates denoted $\gamma_{\text {nrad }}^{p}$ and $\gamma_{\text {nrad }}^{d}$, respectively.

We have measured spontaneous emission decay dynamics of InAs QDs positioned at 28 different distances from a GaAs/air interface, see inset of Fig. 1(b). Further experimental details can be found in Refs. 12 and 15 . We record the number of photons emitted per time interval $N(t)$ from the QDs. Solving the rate equations for the three-level system of Fig. 1(a) results in a biexponential decay $N(t)=A_{f} e^{-\gamma_{f} t}$ $+A_{s} e^{-\gamma_{s} t}$. Here subscripts $s$ and $f$ denote slow and fast decay rates, respectively. Figure 1(b) shows an example of a spontaneous emission decay curve together with a biexponential fit obtained with a fixed background level to account for the measured contribution from dark counts and after pulsing of the detector. Excellent agreement between the experiment and the model is apparent from the weighted residuals reproduced in Fig. 1(c).

The four parameters $\gamma_{f / s}, A_{f / s}$ are obtained by fitting the experimental decay curves. We have $\gamma_{f} \simeq \gamma_{\mathrm{rad}}+\gamma_{\text {nrad }}^{b}$ and $\gamma_{s}$ $\simeq \gamma_{\text {nrad }}^{d}$, where it has been assumed that the spin-flip rate is 

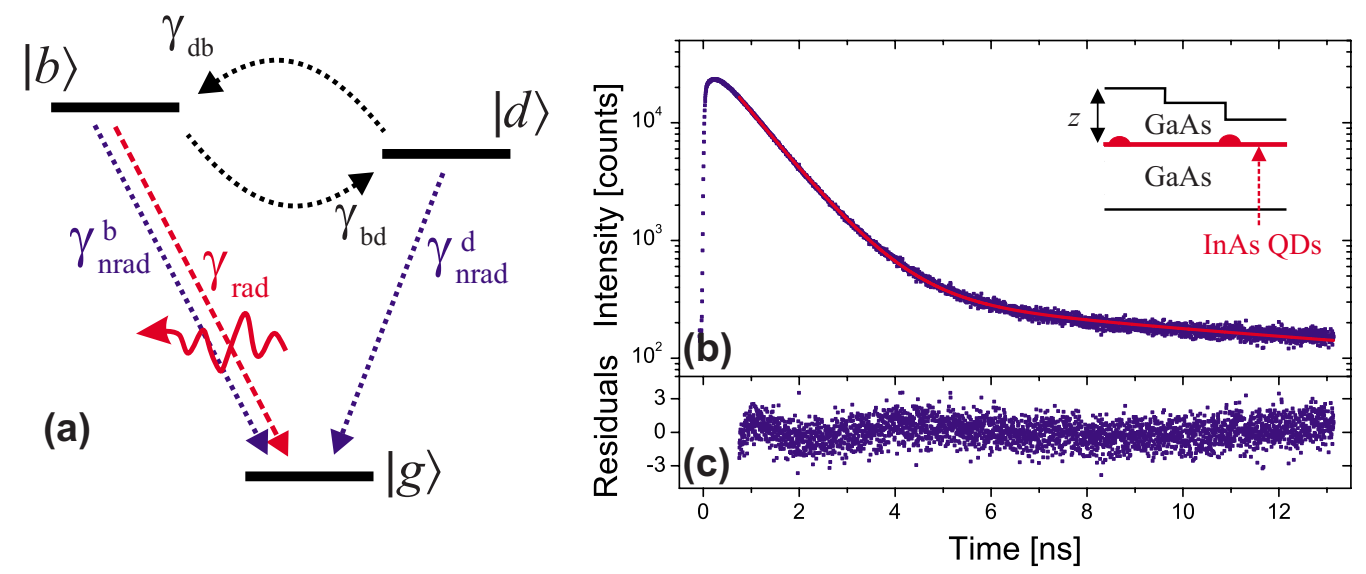

FIG. 1. (Color online) (a) Three-level exciton scheme consisting of a bright $|b\rangle$ and a dark $|d\rangle$ state that are coupled through the spin-flip rates $\gamma_{b d}$ and $\gamma_{d b}$. Radiative recombination $\left(\gamma_{\text {rad }}\right)$ to the ground state $|g\rangle$ (no exciton) is only possible for the bright state, while both the bright $\left(\gamma_{\text {nrad }}^{b}\right)$ and the dark state $\left(\gamma_{\text {nrad }}^{d}\right)$ can decay nonradiatively. (b) Typical decay curve acquired at a detection energy of $1.204 \mathrm{eV}$ (blue squares) at the sample with QDs positioned $170 \mathrm{~nm}$ from the interface. The red line is a fit of the biexponential model to the data. Inset: sketch of the sample showing three different distances between the QDs and the GaAs-air interface. (c) The weighted residual obtained from the biexponential fit to the data.

slow compared to both radiative and nonradiative processes, which is a very good approximation as seen below. Thus, the spin-flip rate cannot be obtained from the measured rates $\gamma_{f / s}$ since they are dominated by other decay contributions. However, the amplitudes $A_{f / s}$ contain additional information and the dark-bright spin-flip rate is contained in their ratio

$$
\frac{A_{f}(z)}{A_{s}(z)}=\frac{\gamma_{f}(z)-\gamma_{s}}{\gamma_{d b}(z)} \frac{\rho_{b}(t=0)}{\rho_{d}(t=0)}-1,
$$

where $\rho_{b}(t=0) / \rho_{d}(t=0)$ is the ratio between the initial populations of bright and dark excitons after an excitation pulse and $z$ is the distance to the interface. The repetitive nature of the experiment implies that the slow amplitude contains contributions from previous excitation pulses, which is accounted for by correcting the amplitudes according to $A_{s}$ $\rightarrow A_{s}\left(1-e^{-\gamma_{s} T}\right)$ where $T=13.5 \mathrm{~ns}$ is the repetition period of the excitation laser. The spin-flip rate is obtained from experimentally determined parameters by

$$
\gamma_{d b}(z)=\frac{\gamma_{f}(z)-\gamma_{s}}{1+A_{f}(z) / A_{s}(z)} \frac{\rho_{b}(t=0)}{\rho_{d}(t=0)} .
$$

It is essential that the ratio of the amplitudes enters in Eq. (2) since the absolute size of $A_{f / s}$ will depend on, e.g., the total collection efficiency of the radiated light or the number of QDs probed. Since the bright and dark excitons recombine radiatively on the same transition [cf. Fig. 1(a)] these dependencies do not contribute to the amplitude ratio. Quite remarkably, assessing the amplitude ratio allows for extraction of spin-flip rates that are slower than the repetition period of the measurement.

In Fig. 2 the slow decay rate and the ratio of the amplitudes are plotted versus distance to the interface. The fast rate (not shown) varies periodically with distance in full agreement with the modified LDOS caused by the reflecting interface. ${ }^{12}$ In contrast, the slow decay rate is constant within the error bars of the measurement, which confirms that it is dominated by nonradiative recombination of dark excitons $\left(\gamma_{\text {nrad }}^{d}\right)$. The average decay rate of $\gamma_{s}=0.097 \pm 0.008 \mathrm{~ns}^{-1}$ matches very well with the nonradiative decay rate of bright excitons at the same emission energy, which is expected since the bright and dark exciton binding energies are very close. $A_{f}(z) / A_{s}(z)$ is expected to vary proportional to the LDOS through $\gamma_{f}(z)$ [cf. Eq. (1)] and indeed clear oscillations with a period matching the LDOS is observed. In ad-

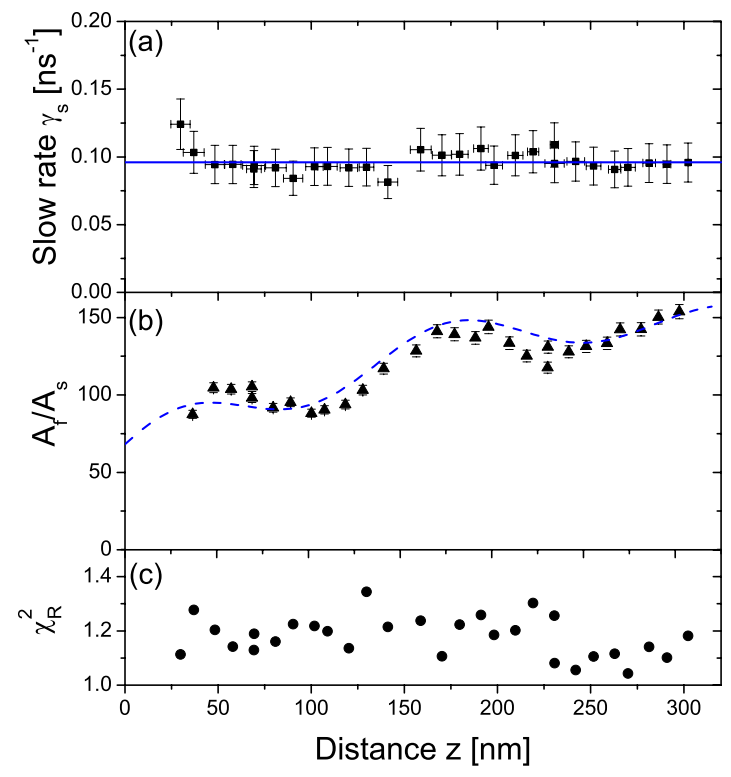

FIG. 2. (Color online) (a) The slow decay rate as a function of distance to the interface measured at $1.204 \mathrm{eV}$. No systematic dependence on distance is observed confirming that the slow rate is due to nonradiative decay of dark excitons. The blue line indicates the averaged rate. (b) Measured ratio of the fast and slow amplitudes versus distance. The dashed blue curve is obtained by multiplying the calculated LDOS with an overall exponential decrease in the spin-flip rate with the distance to the interface. (c) Goodnessof-fit $\left(\chi_{R}^{2}\right)$ for all distances to the interface. 


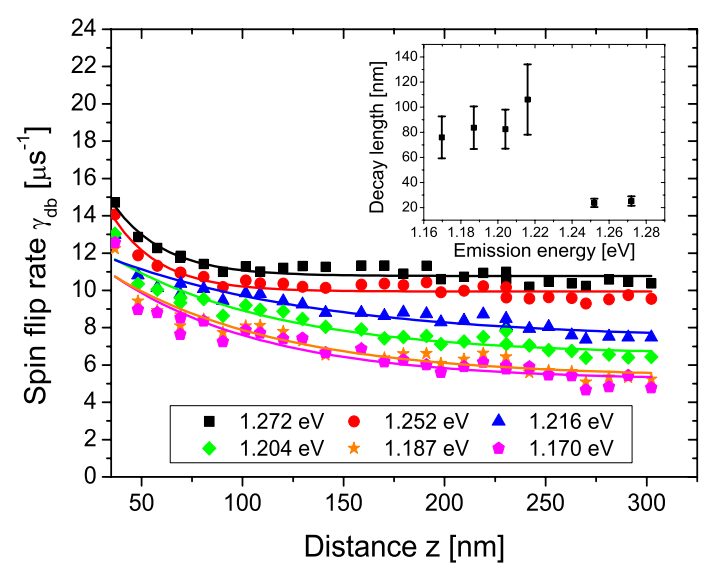

FIG. 3. (Color online) The spin-flip rates versus distance $z$ to the interface for six different emission energies. The plotted curves are fits to the experimental data assuming an exponential decay of the spin-flip rate with the distance to the interface. The inset shows the exponential decay length versus emission energy.

dition the spin-flip rate is found to vary, which gives rise to the overall increase in the amplitude ratio with $z$. Assuming an exponential decrease in the spin-flip rate with $z$, which will be discussed further below, and multiplying by the calculated LDOS we can model the experimental data very well, see Fig. 2(b). The dominating uncertainty in the decay rates stems from variations in the excitation densities from sample to sample, which is expressed by the error bars in Fig. 2. Figure 2(c) shows $\chi_{R}^{2}$ that gauges the quality of the biexponential model for all decay curves. The close proximity to unity confirms the assumed QD model and rules out any significant contributions from additional exciton states, such as charged excitons.

In order to extract the spin-flip rate from Eq. (2), we need to estimate the ratio of the initial populations of bright and dark excitons. Due to the nonresonant excitation, the feeding of the QDs is nongeminate, ${ }^{16}$ thus dark and bright excitons are formed with equal probability. A slight imbalance between the initial populations is nonetheless created due to the finite probability of creating biexcitons. The recombination of biexcitons is dominated by radiative decay, which always leaves behind a bright exciton and thus increase $\rho_{b}(0)$ relative to $\rho_{d}(0)$. The long lifetime of the dark excitons moreover increases the probability of biexciton formation since a residual population of dark excitons is persistent when the subsequent excitation re-excites the QDs. In the present experiment the excitation density was fixed such that on average 0.1 excitons were generated per QD and by solving the rate equations for the QD population including the biexciton level we find $\rho_{b}(t=0) / \rho_{d}(t=0) \approx 1.25$.

The spin-flip rate versus distance to the interface is presented in Fig. 3 for six different emission energies. It is found to vary between 4.7 and $14.7 \mu \mathrm{s}^{-1}$ corresponding to long-lived exciton spin lifetimes between 68 and 215 ns, i.e., two orders of magnitude longer than bright excitons. Surprisingly the spin-flip rate is observed to decay with distance to the interface, which is observed for all emission energies. This dependence is quantitatively described by an exponential decay, and we extract a characteristic decay length vary-

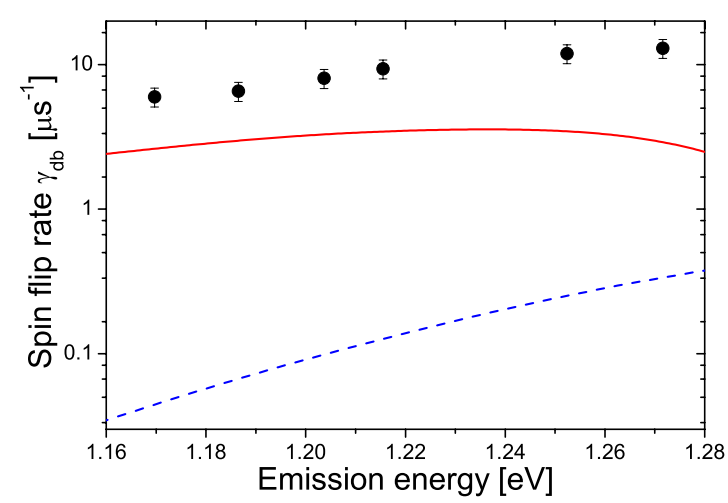

FIG. 4. (Color online) Measured spin-flip rate at different emission energies for the sample with $(z=302 \mathrm{~nm})$ (solid black circles). The theoretically predicted spin-flip rate using the model of Ref. 10 for parameters that reproduce the energy dependence of the radiative decay rate (dashed blue line) and for optimized parameters (solid red line).

ing between 24 and $106 \mathrm{~nm}$ with error bars obtained from the fit of the data therefore expressing the validity of the simple model. The observed increase in the spin-flip rate in the vicinity of the interface could be caused by enhancement of acoustic phonons at the interface. We estimate the phonon wavelength from $\lambda_{\mathrm{ph}}=h v / \Delta_{d b}$, where $h$ is Planck's constant, $v$ is the sound speed, and $\Delta_{d b}$ is the exchange energy splitting between bright and dark states that must be matched by a phonon transition. For GaAs $\lambda_{\mathrm{ph}} \sim 65-85 \mathrm{~nm}$ for longitudinal phonons using the range of exchange energies relevant in the experiment, which matches qualitatively the length scale observed in the experiment. The large decay length and error bars observed for low energies in Fig. 3 suggests that several surface-enhanced phonon modes contribute at low energies, which is not captured by the simple model. Quantitative modeling would require an elaborate theory for the phonon density of states near the interface, which is outside the scope of the present work.

The above results illustrate clearly the importance of nearby surfaces when seeking a quantitative understanding of the dynamics of QDs in nanostructures. Recent examples of intricate surface effects include the increased emission rate observed near a semiconductor interface, ${ }^{15}$ while charge trapping near surfaces was suggested as the mechanism responsible for the surprisingly large QD-nanocavity coupling efficiency at very large detunings observed under nonresonant excitation. ${ }^{17-19}$ These are examples of the new physics found in solid-state implementations of quantum electrodynamics experiments.

Let us now consider the energy dependence of the spinflip rate, which allows to assess how the spin-flip process varies with QD size. In order to minimize the effect of the interface, we plot in Fig. 4 the spin-flip rate versus energy for the sample with the largest QD-interface distance ( $z$ $=302 \mathrm{~nm}$ ). The spin-flip rate is observed to increase significantly with the emission energy and varies from $6 \mathrm{~ns}^{-1}$ at $1.170 \mathrm{eV}$ to $13 \mathrm{~ns}^{-1}$ at $1.272 \mathrm{eV}$ meaning that the characteristic spin-flip time can be prolonged by more than a factor of two by varying the QD size.

The energy dependence of the spin-flip rate can be com- 
pared to theory using the model introduced in Ref. 10. In this work the dark-bright exciton spin-flip rate was calculated due to the combined effect of short-range exchange interaction and acoustic phonons. A complex behavior is expected since the spin-flip rate is predicted to depend on the energy splitting between the lowest and first excited exciton states $\Delta_{12}$, the exchange energy splitting $\Delta_{d b}$, as well as the size of the electron and hole wave functions.

The experimental data for the energy-dependent spin-flip rates are compared to theory using experimentally realistic parameters: by recording the emission spectrum of the QDs at high pump power where the ground state is saturated, we obtain $\Delta_{12}=120 \pm 20 \mathrm{meV}$. The exchange splitting depends strongly on the indium mole fraction, ${ }^{20}$ and it is enhanced in QDs due to the strong localization of the electron and hole wave functions as described by the enhancement factor. ${ }^{21}$ Furthermore, the spin-flip rate depends on the electron and hole wave functions through a form factor. ${ }^{22}$ We have calculated the electron and hole wave functions using the theory and parameters of Ref. 15. All other parameters are taken from Ref. 10 and the references given above.

The comparison to theory is presented in Fig. 4. The dashed blue curve is for parameters that reproduce the frequency dependence of the radiative decay rate ${ }^{15}$ (aspect ratio: $1 / 2$, indium mole fraction: 0.46 , QD heights: between 4 and $5.8 \mathrm{~nm}$ ). The observed increase in the spin-flip rate with energy is reproduced by the theory. However, the calculated rates are two orders of magnitude smaller than the measured values. We have explored the origin of this deviation by optimizing the model parameters in order to maximize the spin- flip rate, see the solid red curve in Fig. 4 (aspect ratio: 1/3, indium mole fraction: 0.90, QD heights: between 1.9 and 2.4 $\mathrm{nm})$. In this case the predicted spin-flip rates approach the measured rates although a systematic discrepancy is still observed. Furthermore, a rather weak frequency dependence of the spin-flip rate is predicted since the finite confinement potentials impose lower limits to the achievable compression of the wave functions. We conclude that additional spin-flip processes must be included in a complete theory. Additional contributions may arise due to spin-orbit coupling ${ }^{11}$ or longrange exchange effects. ${ }^{23}$

In conclusion, we have measured the spin-flip rate from dark to bright excitons using time-resolved fluorescence spectroscopy. The spin-flip rate increases significantly when approaching the sample surface suggesting an enhancement due to surface acoustic phonons. The energy dependence of the spin-flip rate was compared to a recent theory, where the spin flip is induced by short-range exchange interaction between electrons and holes while the necessary energy is provided by acoustic phonons. Our data illustrate that additional processes contribute to exciton spin flips in QDs. In addition, the response of QDs were found to be strongly influenced by the dark states of significant importance for quantitative experiments on light-matter interaction in QD based optical devices.

We gratefully acknowledge the Danish Research Agency for financial support (Projects No. FNU 272-05-0083, No. 272-06-0138, and No. FTP 274-07-0459). *pelo@fotonik.dtu.dk

†http://www.fotonik.dtu.dk/quantumphotonics

${ }^{1}$ A. Imamoğlu, D. D. Awschalom, G. Burkard, D. P. DiVincenzo, D. Loss, M. Sherwin, and A. Small, Phys. Rev. Lett. 83, 4204 (1999).

${ }^{2}$ O. Labeau, P. Tamarat, and B. Lounis, Phys. Rev. Lett. 90, 257404 (2003).

${ }^{3}$ J. M. Smith, P. A. Dalgarno, R. J. Warburton, A. O. Govorov, K. Karrai, B. D. Gerardot, and P. M. Petroff, Phys. Rev. Lett. 94, 197402 (2005).

${ }^{4}$ B. Lounis and M. Orrit, Rep. Prog. Phys. 68, 1129 (2005).

${ }^{5}$ S. Strauf et al., Nat. Photonics 1, 704 (2007).

${ }^{6}$ T. Lund-Hansen, S. Stobbe, B. Julsgaard, H. Thyrrestrup, T. Sünner, M. Kamp, A. Forchel, and P. Lodahl, Phys. Rev. Lett. 101, 113903 (2008).

${ }^{7}$ M. Reischle, G. J. Beirne, R. Roßbach, M. Jetter, and P. Michler, Phys. Rev. Lett. 101, 146402 (2008).

${ }^{8}$ P. Lodahl et al., Nature (London) 430, 654 (2004).

${ }^{9}$ B. Julsgaard et al., Appl. Phys. Lett. 93, 094102 (2008).

${ }^{10}$ K. Roszak, V. M. Axt, T. Kuhn, and P. Machnikowski, Phys. Rev. B 76, 195324 (2007); 77, 249905(E) (2008).

${ }^{11}$ E. Tsitsishvili, R. v. Baltz, and H. Kalt, Phys. Rev. B 72, 155333 (2005).
${ }^{12}$ J. Johansen, S. Stobbe, I. S. Nikolaev, T. Lund-Hansen, P. T. Kristensen, J. M. Hvam, W. L. Vos, and P. Lodahl, Phys. Rev. B 77, 073303 (2008).

${ }^{13}$ M. Winger, A. Badolato, K. J. Hennessy, E. L. Hu, and A. Imamoğlu, Phys. Rev. Lett. 101, 226808 (2008).

${ }^{14}$ M. Bayer et al., Phys. Rev. B 65, 195315 (2002).

${ }^{15}$ S. Stobbe, J. Johansen, P. T. Kristensen, J. M. Hvam, and P. Lodahl, Phys. Rev. B 80, 155307 (2009).

${ }^{16}$ B. Baylac et al., Surf. Sci. 326, 161 (1995).

${ }^{17}$ K. Hennessy et al., Nature (London) 445, 896 (2007).

${ }^{18}$ M. Kaniber, A. Laucht, A. Neumann, J. M. Villas-Bôas, M. Bichler, M.-C. Amann, and J. J. Finley, Phys. Rev. B 77, 161303(R) (2008).

${ }^{19}$ A. Laucht et al., New J. Phys. 11, 023034 (2009).

${ }^{20}$ H. Fu, L.-W. Wang, and A. Zunger, Phys. Rev. B 59, 5568 (1999).

${ }^{21}$ R. Romestain and G. Fishman, Phys. Rev. B 49, 1774 (1994).

${ }^{22}$ A. Grodecka, L. Jacak, P. Machnikowski, and K. Roszak, in Quantum Dots: Research Developments, edited by P. A. Ling (Nova Science, New York, 2005), p. 47.

${ }^{23}$ J. W. Luo, A. Franceschetti, and A. Zunger, Phys. Rev. B 79, 201301(R) (2009). 